\title{
UNE NOUVELLE ESPÈCE DE Modiolopsis (BIVALVIA) DANS L'ARENIG (ORDOVICIEN INFÉRIEUR) DE DAROCA (ARAGON, ESPAGNE); RÉFLEXIONS SUR LA DENTURE DES BIVALVES PRIMITIFS
}

\author{
Claude BABIN' et Wolfgang HAMMANN² \\ ' UER Sciences de la Terre, Université Claude Bernard-Lyon I, 27-43, bd du \\ 11 novembre, 69622 Villeurbanne Cedex, France \\ 2 Ahornstrasse 3, 97535 Wasserlosen, Allemagne
}

Babin C. et Hammann W. 2001. Une nouvelle espèce de Modiolopsis (Bivalvia) dans l'Arenig (Ordovicien inférieur) de Daroca (Aragon, Espagne); réflexions sur la denture des bivalves primitifs. [A new species of Modiolopsis (Bivalvia) from the Arenig (Early Ordovician) of Daroca (Aragon, Spain); thoughts on the dentition of the primitive bivalves.] Revista Espainola de Paleontología, 16(2), 269-282. ISSN 0213-6937.

\begin{abstract}
A new species of Modiolopsis, M. aragonensis, is described from the middle or upper Armorican Sandstone (Arenig, Lower Ordovician) of the area around Daroca (Aragon, Spain). The study of this edentulous genus, the presence of which is thus confirmed in the Lower Ordovician, presents the opportunity to develop some thoughts on the phyletic relations of the primitive bivalves taking their types of dentition into consideration. The comparison of the recently described genera from the Cambrian and the Lower Ordovician apparently shows a rapid prevalence of various palaeoheterodonts. Consequently it can be suggested that the hypothetical archetype unknown and named proterodont could have been edentulous or with a very primitive dentition of undefined type. From such an ancestor which would be probably found in the lowermost Cambrian (Nemkyt-Daldynian) or in the uppermost Proterozoic (Vendian), the palaeotaxodont and palaeoheterodont types could have been sister groups with a rapid diversification of the latter.
\end{abstract}

Keywords: Bivalves, systematics, Cambrian, Lower Ordovician, Spain, evolution.

\section{RÉSUMÉ}

Une espèce nouvelle de Modiolopsis, M. aragonensis, est décrite dans le Grès armoricain, moyen ou supérieur (Arenig, Ordovicien inférieur) des environs de Daroca (Aragon, Espagne). L'examen de ce genre édentule, dont la présence dans l'Ordovicien inférieur se trouve ainsi confirmée, introduit à quelques réflexions générales sur les relations phylétiques des bivalves primitifs d'après la considération de leurs types de dentures. La comparaison des genres récemment décrits dans le Cambrien et dans l'Ordovicien inférieur paraît montrer une rapide prééminence de paléohétérodontes diversifiés. Cela peut suggérer que l'archétype hypothétique, inconnu et nommé protérodonte, pourrait avoir été édentule ou à denture très fruste et de type indéterminé. A partir d'un tel ancêtre, à rechercher peut-être dans le Cambrien basal (Nemkyt-Daldynien) ou le Protérozoïque terminal (Vendien), les types paléotaxodonte et paléohétérodonte se seraient individualisés comme des groupes-frères avec une rapide diversification des paléohétérodontes.

Mots-clés: Bivalves, systématique, Cambrien, Ordovicien inférieur, Espagne, évolution.

\section{RESUMEN}

Se describe una nueva especie de Modiolopsis, M. aragonensis, de la parte media o superior de la Cuarcita Armoricana (Arenig, Ordovícico Inferior) cerca de Daroca (Aragón, España). El estudio de este género edéntulo, cuya presencia en el Ordovícico Inferior resulta confirmada, permite hacer reflexiones generales sobre las relaciones filéticas de los bivalvos primitivos considerando sus tipos de denticiones. La comparación de los géneros descritos recientemente del Cámbrico y del Ordovícico Inferior parece indicar una rápida preeminencia de los paleoheterodontos diversificados. Eso puede sugerir que el arquetipo hipotético, desconocido y llamado 
proterodonto, podría haber sido edéntulo o con una dentición muy sencilla de tipo indeterminado. A partir de un tal antecesor, que se podría buscar en la parte más baja del Cámbrico (Nemkyt-Daldyniense) o en el Proterozoico terminal (Vendiense), los tipos paleotaxodonto y paleoheterodonto se habrían individualizado como gruposhermanos con una rápida diversificación de los paleoheterodontos.

Palabras clave: Bivalvos, sistemática, Cámbrico, Ordovícico Inferior, España, evolución.

\section{INTRODUCTION}

La Formation du Grès armoricain, initialement définie par Rouault (1850) en Bretagne est, pour l'essentiel, selon Paris (1990), de l'Arenig moyen (Whitlandien sensu Fortey et Owens, 1987). Il y présente typiquement une composition tripartite, Grès armoricain inférieur, "Schistes intermédiaires" différemment désignés selon les régions du Massif armoricain, Grès armoricain supérieur. C'est dans ce dernier membre qu'ont été récoltés, dès le $19^{\mathrm{e}}$ siècle, des mollusques bivalves décrits par Barrois (1891) puis révisés par Babin (1966). Ces faunes avaient été recueillies dans les Synclinaux du sud de Rennes et sur le flanc nord du Synclinal d'Angers. Leur état de conservation, très médiocre, en rendait l'étude systématique malaisée comme le remarquait Barrois (1891). Cet auteur décrivit pourtant vingt-huit espèces réparties entre quatorze genres. Beaucoup de ces spécimens demeurent, en réalité, indéterminables et la diversité réelle de cette faune reste mal établie. Il convient, néanmoins, de signaler que Barrois cita et figura deux espèces de Modiolopsis. Il détermina $M$. cailliaudi de Tromelin et Lebesconte, 1875 un exemplaire très partiel, déposé au Musée d'Histoire naturelle de Nantes, qui est le seul spécimen connu pour cette espèce, ce qui incite à limiter la désignation spécifique à cet unique représentant dont l'attribution générique ellemême demeure mal assurée. $M$. davyi Barrois, 1891 est certainement, en revanche, un modiolopside.

D'autres modiolopsides peuvent être répertoriés dans l'Arenig de diverses régions. Dans la Montagne Noire (sud de la France), Babin (1982) a figuré un petit Modiolopsis sp., exemplaire unique provenant de la Formation du Foulon datée de l'Arenig inférieur (Paris et al., 2000). En Espagne occidentale (Province de Cáceres) sur la base d'un exemplaire unique également, Modiolopsis sp. est cité dans le Grès armoricain supérieur (Gutiérrez-Marco et al., 1997). Au Pays de Galles, Cope (1996b) a décrit dans l'Arenig inférieur (Moridunien) deux formes désignées Modiolopsis sp. A et $M$. sp. B connues chacune par deux spécimens seulement. Dans l'Arenig d'Argentine, Harrington (1938) a décrit Modiolopsis sanbernardica; la figuration qu'en donne l'auteur (pl. 2, fig. 26) de l'exemplaire unique et à la charnière inobservable n'est pas entièrement convaincante; ce spécimen n'a pas été revu depuis. Cependant, selon T. Sánchez (e-mail du 19 octobre 2000), le genre est probablement présent dans l'Arenig de la Précordillère d'Argentine mais il est spécifiquement indéterminable.

Comme on le constate, si la présence du genre Modiolopsis se trouve attestée dès 1'Arenig basal, il semble avoir été néanmoins peu fréquent dans l'ensemble de l'Ordovicien inférieur. Cela confère un intérêt certain au matériel provenant de l'Aragon.

\section{SITUATION GÉOGRAPHIQUE ET DONNEES STRATIGRAPHIQUES \\ par Wolfgang Hammann}

La localité fossilifère étudiée est située à quinze kilomètres à l'ouest de Daroca dans la Chaîne ibérique occidentale qui constitue le flanc sud-ouest du massif paléozoïque de la Chaîne ibérique aragonaise (Fig. 1). Dans cette région affleure une succession cambroordovicienne complète qui plonge relativement régulièrement vers le sud-ouest. Au sud et au sud-ouest de Daroca, la Formation du Grès armoricain et des formations gréseuses, très réduites, du Llanvirn-Caradoc constituent la bordure du Paléozoïque et disparaissent sous la couverture des sédiments mésozoïques et quaternaires.

La Formation du Grès armoricain de la Chaîne ibérique a une puissance d'environ $600 \mathrm{~m}$ et est subdivisée en trois membres (Carls, 1975, p. 133; Wolf, 1980, p. 130). Les membres inférieur et supérieur sont caractérisés par la prévalence des quartzites purs en gros bancs tandis que le membre moyen est constitué par une alternance de siltites et de grès impurs. Dans les Chaînes ibériques occidentales, le membre supérieur renferme deux intercalations silteuses importantes qui offrent des ressemblances lithologiques avec le membre moyen.

Dans les Chaînes ibériques, l'âge stratigraphique précis de la formation n'a pas encore été bien établi. La présence de Dinobolus cf. brimonti Rouault, 1850 dans le membre inférieur (Wiemer, 1975) et l'existence d'un ichnofacies à Cruziana rugosa permet une datation approchée en Arenig. En effet, la Formation Santed sousjacente ayant livré une faune avec Megistaspis (Ekeraspis) filacovi (Munier-Chalmas et Bergeron, 1888) indicateur, dans la Montagne Noire, de l'Arenig inférieur, la base du Grès armoricain devait se trouver plus haut (Wolf, 1980, p. 128, 129; Hammann et al., 1982, p. 33). L'âge du sommet de la formation est encore plus difficile à estimer parce qu'elle est, dans la Chaîne ibérique occidentale, séparée par une lacune des grès ferrugineux superposés qui livrent des graptolites du Llanvirn de la Zone à Didymograptus murchisoni (Gutiérrez-Marco, 1986, p. 76).

Les premiers restes fossiles ont été découverts dans des cailloux volants lors de travaux cartographiques par Wiemer (1975, p. 55) à 4,5 km à l'est du village de Cubel sur le versant NE d'une élévation $(1.300 \mathrm{~m})$ largement couverte par une végétation méditerranéenne. La couche 


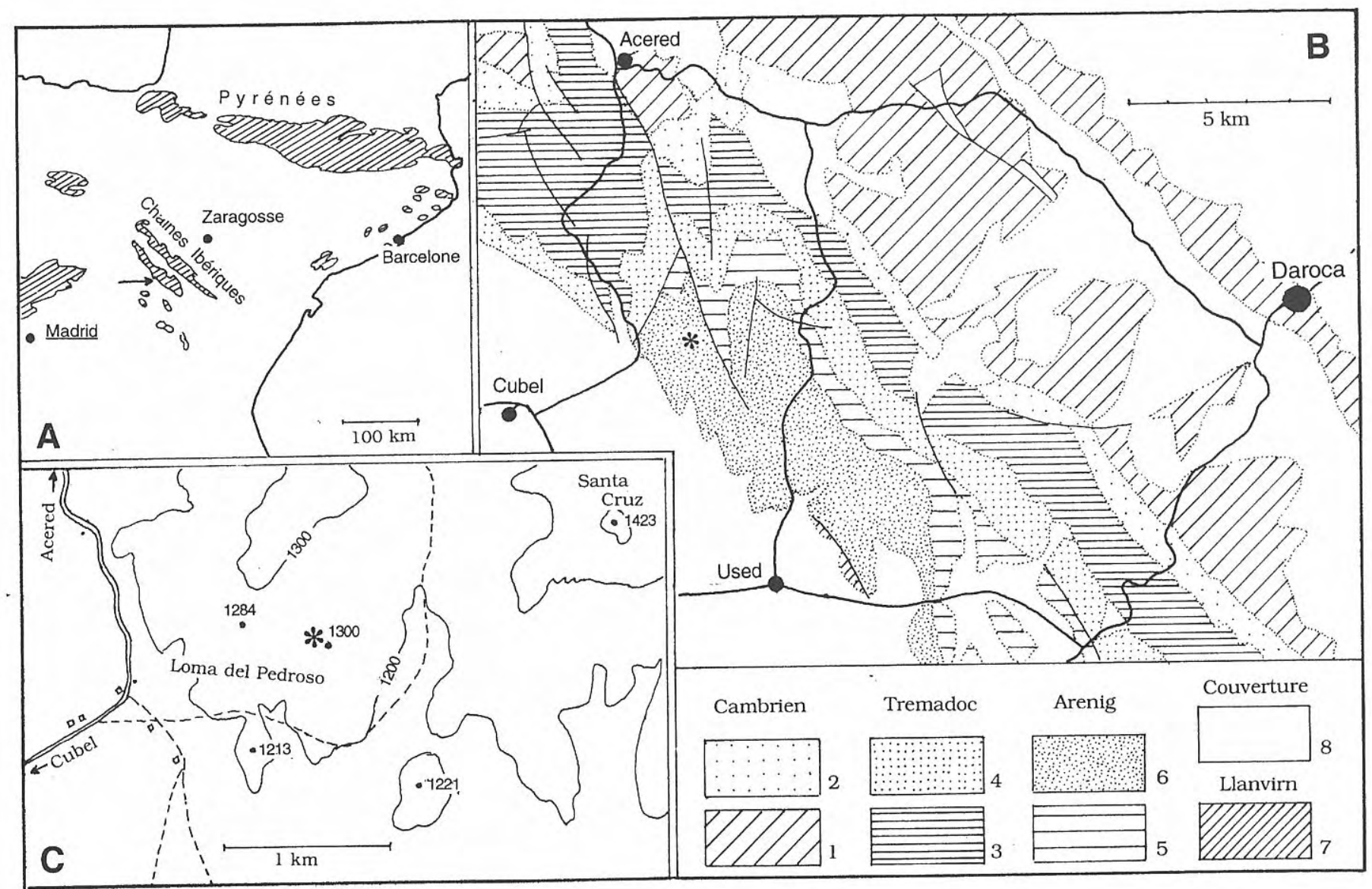

Figure 1. Localisation du gisement. A. Les massifs hercyniens dans le nord-est de l'Espagne comprenant les Chaînes ibériques; la flèche indique la zone décrite dans la Chaîne ibérique occidentale. B. Carte géologique de la région entre Daroca et Cubel. 1. Cambrien inférieur à supérieur indifférencié. 2. Formation de Valconchán (Cambrien supérieur à ?Tremadoc). 3. Formation Borrachón (Tremadoc). 4. Formation Deré (Tremadoc). 5. Formation Santed (Arenig inférieur). 6. Formation du Grès armoricain (Arenig moyen). 7. Llanvirn-Caradoc. 8. Couverture posthercynienne (Trias-Quaternaire). C. Carte topographique des environs du gisement fossilifère qui est indiqué par un astérisque.

Location of the outcrop. A. The hercynian massifs in the northeast of Spain including the Iberian Chains; the arrow shows the described zone of the western Iberian Chain. B. Geological map of the area between Daroca and Cubel. 1. Undifferentiated Lower to Upper Cambrian. 2. Valconchán Formation (Upper Cambrian to ?Tremadoc). 3. Borrachón Formation (Tremadoc). 4. Deré Formation (Tremadoc). 5. Santed Formation (Lower Arenig). 6. Armorican Sandstone Formation (Middle Arenig). 7. Llanvirn-Caradoc. 8. Posthercynian (Triassic-Quaternary) cover. C. Topographical map of the area around the fossiliferous outcrop marked by an asterisk.

fossilifère a été trouvée et exploitée provisoirement dans une tranchée creusée en 1978 par W. Hammann, R. Wolf et S. Kolb (Wolf, 1980, p. 131). De nouvelles fouilles, exécutées depuis 1997, ont considérablement augmenté le nombre des exemplaires bien conservés. De nouveaux travaux sont prévus.

La faune se compose principalement de divers brachiopodes inarticulés (Dinobolus, Lingulepis, Tomasina, etc., en cours d'étude par E. Villas et Z. Herrera à l'Université de Saragosse) et de nombreux exemplaires du bivalve Modiolopsis décrits ici. Moins fréquents, des arthropodes sont représentés par une seule espèce de trilobite, Asaphellus sp., et par des restes fragmentaires d'un genre probablement nouveau de xiphosuride. De plus, quelques exemplaires de conularides et de gastéropodes (Palaeacmea sp.) ont été recueillis. Les schistes intermédiaires ont été échantillonnés pour la recherche de microfossiles organiques.

Du point de vue stratigraphique, la situation du gisement est encore mal établie. La proximité d'une faille et les mauvaises conditions d'affleurement rendent difficile l'attribution précise à l'un des membres du Grès armoricain. Selon Wiemer (1975, p. 80), il s'agit d'une partie du membre supérieur, mais la lithologie ressemble aussi à celle du membre moyen.

Actuellement dans l'affleurement un mètre de la séquence est exposé, présentant une alternance de siltites et argilites avec deux bancs gréseux fossilifères intercalés. Ces deux couches, respectivement de 10 et 15$20 \mathrm{~cm}$ d'épaisseur, sont formées de sables purs, bien lavés avec galets mous à la base. La surface de base du banc inférieur, le plus épais et le plus fossilifère, est 
couverte de tool marks bien alignés qui proviennent probablement du transport des restes squelettiques par des courants. La fragmentation des fossiles augmente avec leur taille. Tandis que les tagmata du grand xiphosuride sont toujours fragmentés, la plupart des lingules sont restées complètes. De taille intermédiaire, les restes désarticulés des bivalves et des trilobites sont tantôt complets, tantôt brisés. Les deux couches gréseuses fossilifères peuvent être interprétées comme des "storm channel sands" (Seilacher, 1982) représentant des événements de tempête dans un milieu littoral.

\section{ETUDE PALÉONTOLOGIQUE} par Claude Babin

La classification des bivalves utilisée par les paléontologues a été très mouvante au cours des dernières décennies. Ils ont été généralement répartis entre plusieurs sous-classes (voir, par exemple, Newell in Moore, 1969, p. N205-224 ; Pojeta, 1987; Cope, 1996a, b, 1997). Dans sa récente synopsis, Amler (1999) accorde statut de superordres à plusieurs de ces sous-classes et les répartit entre deux infra-classes, Protobranchia et Autobranchia. Cette division binaire, mais avec statut de sous-classes, qui correspond à celles des zoologistes, paraît être désormais le plus souvent adoptée en paléontologie (Waller, 1990, 1998; Cope, 2000; Carter et al., 2000); elle est celle retenue ici. Cope (2000) et Carter et al. (2000) utilisent le terme Autolamellibranchiata pour désigner la seconde de ces sous-classes parce qu'il est celui originellement proposé par Grobben (1894); je préfère néanmoins, émendant ce terme initial, conserver celui d'Autobranchia, entériné par l'usage et moins ambigu puisqu'il inclut à la fois filibranches, eulamellibranches et septibranches.

Par ailleurs, pour divers auteurs il convient de dissocier, au sein de l'ordre Modiomorphoida, les familles Modiomorphidae et Modiolopsidae. Dès 1971, Pojeta (p. 20) suggéra cette séparation, ces deux familles seront alors placées dans l'ordre Mytiloida et dans la sous-classe Isofilibranchia. Liljedahl (1994) retient cette distinction, fondée sur la denture, en la plaçant au niveau infrafamilial seulement avec les sous-familles Modiomorphinae (présence de dents) et Modiolopsinae (charnière édentule) dans la famille Modiomorphidae qui reste placée dans les mytiloïdes et les isofilibranches. Bradshaw (1999) utilise l'ordre Modiomorphoida qu'elle maintient aussi dans la sous-classe Isofilibranchia. Au contraire, Cope (1996b), qui adopte la séparation des deux familles, place l'ordre Modiomorphoida au sein des Palaeoheterodonta.

Pour Fang et Morris (1997) et Fang (1998), en revanche, la famille Modiomorphidae (dont devient synonyme celle des Permophoridae) prend place dans l'ordre Pholadomyoida au sein de la sous-classe Anomalodesmata, position déjà suggérée par Waller (1990) puis admise par Johnston $(1993,1996)$ et par Carter et al. (2000). Simultanément, la famille Modiolopsidae à charnière édentule ainsi qu'une nouvelle famille, Modiolodontidae à petites dents cardinales, sont placées dans l'ordre Mytiloida au sein de la sous-classe Pteriomorphia. De telles affinités sont corroborées, selon Carter et Seed (1998, p. 88) par les caractères ligamentaires. Cope (2000) et Carter et al. (2000) adoptent finalement cette classification également retenue ici. Notons cependant que ces nombreuses divergences et fluctuations reflètent clairement les difficultés d'interprétation de ces groupes primitifs de bivalves.

Le matériel étudié est déposé dans les collections du Museo del Área de Paleontología de la Universidad de Zaragoza (sigle : MPZ).

\section{CLASSE BIVALVIA Linné, 1758 SOUS-CLASSE AUTOBRANCHIA Grobben, 1894 SUPERORDRE PTERIOMORPHIA Beurlen, 1944 ORDRE MYTILOIDA Ferussac, 1822 Famille Modiolopsidae Fischer, 1887}

Genre Modiolopsis Hall, 1847

Espèce-type : Pterinea modiolaris Conrad, 1838 par désignation originale de Hall, 1847.

Coquille équivalve, très inéquilatérale; crochet en position très antérieure; extrémité postérieure élargie; ornementation concentrique; impression adductrice antérieure plus ou moins imprimée dans la valve, mais parfois indistincte; ligne palléale entière; ligament externe, opisthodète; charnière édentule.

Le caractère édentule de la charnière constitue un caractère diagnostique du genre. Les discussions anciennes (voir Babin, 1966, p. 184; Pojeta, 1971, p. 20) concernant la denture paraissent devoir être définitivement closes. Si le contour, parfois dit modioliforme, se retrouve, par convergence, chez d'autres genres éloignés, tel l'anomalodesmate Modiomorpha notamment, l'absence d'un sulcus umbono-ventral et d'une sinuosité du bord palléal retenue comme un autre caractère générique de Modiolopsis par LaRocque et Newell (in Moore, 1969, p. N393-N399) n'est peut-être pas générale (voir, par exemple, la forme désignée Modiolopsis sp. in Babin, 1982, pl. 10, fig. 14, 15).

Ainsi défini, le genre Modiolopsis n'est connu que de l'Ordovicien; peu commun à l'Arenig semble-t-il, il paraît avoir été parfois abondant à l'Ordovicien supérieur.

\section{Modiolopsis aragonensis nov. sp.} Fig. 2

Holotype: Moule interne d'une valve gauche, MPZ 01/1.

Paratypes: Moules internes de deux valves droites (MPZ 01/2, MPZ 01/3) et d'une valve gauche (MPZ 01/4).

Localité-type: Loma de Pedroso à l'ouest du village de Cubel.

Strate-type: Membre moyen ou supérieur (?) de la Formation des Grès armoricains, Arenig (probablement moyen). 



Figure 2. Modiolopsis aragonensis nov. sp. a, f. Paratype, MPZ 01/2. Moule interne d'une valve droite en vue latérale (a) et cardinale (f) qui montre la charnière édentule. b, c. Holotype, MPZ 01/1. Moule interne d'une valve gauche; la vue latérale (b) montre une petite empreinte ovale qui pourrait être celle de l'adducteur antérieur; la vue cardinale (c) montre que la charnière est parfaitement édentule. d. Paratype, MPZ 01/3. Moule interne d'une valve droite dont le talus post-carénal porte une trace ovale (empreinte de l'adducteur postérieur ou artefact?). e. Paratype, MPZ 01/4. Moule interne d'une valve gauche portant peut-être une trace de l'empreinte adductrice antérieure. Tous les exemplaires x1,9.

Modiolopsis aragonensis nov. sp. a, $f$. Paratype, MPZ 01/2. Internal mould of a right valve, lateral view (a), cardinal view $(f)$ showing the edentulous hinge. $b, c$. Holotype, MPZ 01/1. Internal mould of a left valve; the lateral view $(b)$ shows a small oval impression which could be the anterior adductor one; the cardinal view (c) shows a perfectly edentulous hinge. d. Paratype, MPZ 01/3. Internal mould of a right valve the postcarinal slope of which shows an oval impression (posterior adductor or artifact?). e. Paratype, MPZ 01/4. Internal mould of a left valve showing maybe the anterior adductor scar. All specimens x1.9.

Origine du nom: De la Communauté autonome d'Aragon (Espagne)

Matériel: 78 spécimens (MPZ 01/1-MPZ 01/78) dont l'holotype; sous forme de moules internes et/ou externes de valves isolées, 43 valves gauches et 35 valves droites. 11 spécimens sont des valves entières dont l'état est instructif; 23 sont des valves entières en médiocre état; 44 moules très partiels correspondent essentiellement à des parties postérieures de valves (41 pour 3 parties antérieures).

\section{Diagnose}

Une espèce de Modiolopsis assez fortement carénée, à extrémité postéro-ventrale légèrement étirée, sans empreintes adductrices marquées.
A species of Modiolopsis with a quite strong carina, a fairly drawn out posteroventral end, and without distinct adductor scars.

\section{Description et comparaisons}

La coquille modioliforme se caractérise par la présence d'une carène mousse s'étendant de l'umbo à l'angle postéroventral qui est souvent légèrement étiré. Cette carène détermine un talus vers le bord cardinal rectiligne. Le crochet, peu développé, est très antérieur, placé vers le dixième ou le neuvième antérieur de la longueur de la coquille. Le bord antérieur, peu élevé, est arrondi et se raccorde sans discontinuité au bord palléal rectiligne, il est plus rarement marqué d'une très discrète concavité malgré toute absence de sulcus. Le bord postérieur, arrondi, étiré 
dans sa partie ventrale, est oblique et rectiligne pour se raccorder à la ligne cardinale suivant un angle très obtus. La coquille est peu convexe; son ornementation, peu conservée, est faite de côtes concentriques mal définies.

Les empreintes des muscles adducteurs sont généralement indiscernables. Seuls deux spécimens (l'holotype et MPZ 01/4) portent une petite empreinte ovale qui peut correspondre à celle de 1'adducteur antérieur (Fig. 2 b, e). Un autre exemplaire (MPZ 01/3) offre une trace ovale sur le talus postérieur qui marquerait, si elle n'est pas un artefact, l'emplacement de l'adducteur postérieur (Fig. 2, d).

La charnière, dégagée sur quelques spécimens, est parfaitement dépourvue de dents (Fig. 2, c, f).

Par le contour, la forme de la carène et l'obsolescence de l'empreinte adductrice antérieure, les spécimens aragonais diffèrent de ceux de l'Arenig gallois figurés par Cope (1996b, pl. 3, fig. 7 et pl. 6, fig. 7) et des diverses espèces décrites dans l'Ordovicien supérieur du Massif armoricain (Babin, 1966), d'Amérique du Nord (Pojeta, 1971), de Scandinavie (Isberg, 1934), du Kazakhstan (Khalfin, 1958) ou de Sibérie (Krasilova, 1979). La nouvelle espèce n'inclut probablement pas l'exemplaire unique décrit et figuré comme Modiolopsis sp. par Gutiérrez-Marco et al. (1997) provenant également du Quartzite armoricain supérieur d'Espagne mais situé plus à l'ouest [gisement de Alía (Cáceres) sur le flanc NE du synclinal de Guadarranque]. Ce spécimen, subcontemporain, n'offre pas la carène mousse caractéristique de nos exemplaires et son contour postérieur est différent; il demeure que ce moule médiocrement conservé pourrait néanmoins correspondre à un variant individuel.

\section{RÉFLEXIONS SUR LES DENTURES DES BIVALVES PRIMITIFS}

La présence de cette nouvelle espèce de Modiolopsis dans l'Arenig moyen d'Espagne vient compléter l'inventaire qui montre le rôle déterminant qu'assurèrent les plates-formes périgondwaniennes dans la diversification des bivalves durant 1'Ordovicien inférieur (Babin, 1993a, 1995; Cope et Babin, 1999).

Simultanément, l'ancienneté confirmée dans l'Ordovicien inférieur de Modiolopsis avec son type de charnière extrêmement simple, puisque dépourvue de dents, repose le problème du critère denture pour l'interprétation des relations phylogénétiques entre les divers groupes de bivalves primitifs. La documentation disponible a été sensiblement enrichie durant les trois dernières décennies, elle n'apporte pourtant pas, semblet-il, de réponse définitive aux relations phylétiques entre les premiers types de dentures, paléotaxodonte et actinodonte.

Il convient de rappeler à ce sujet que les fossiles les plus anciens considérés comme des mollusques bivalves, Fordilla et Pojetaia, proviennent du Cambrien inférieur.

Fordilla connu en Amérique du Nord, au Groënland, en Sibérie, a été intitialement interprété comme un paléohétérodonte cycloconchide (Pojeta et al., 1973;
Pojeta et Runnegar, 1974; Pojeta, 1975). Plus tard, Fordilla a été considéré comme un isofilibranche modiolopside primitif (Runnegar et Bentley, 1983, Pojeta et Runnegar, 1985). D'autres citations du genre Fordilla ont été faites. Dans le Cambrien d'Espagne, Sampelayo (1935, p. 495) a cité et figuré F. marini (pl. 8, fig.2) qui s'avère être, en réalité, un brachiopode inarticulé attribué au genre Micromitra (Mergl et Liñan, 1986). Dans le Cambrien inférieur de Normandie (France), Doré (1994) a mentionné Fordilla sp.; la révision de ces spécimens, de conservation très médiocre, m'a montré qu'il ne s'agit pas de Fordilla mais probablement de rostroconches du genre Heraultipegma.

Pojetaia, connu en Australie, en Chine, au Danemark (île de Bornholm) et au Maroc, fut initialement placé par son inventeur (Jell, 1980) dans la famille Fordillidae et dans la sous-classe Heteroconchia. Il a, depuis, été interprété comme un paléotaxodonte primitif (Runnegar et Bentley, 1983; Pojeta et Runnegar, 1985).

Ces rapprochements de Fordilla avec les isofilibranches et de Pojetaia avec les paléotaxodontes ont été initialement fondés surtout sur certaines similitudes des contours des coquilles; ce critère morphologique a, en réalité, une signification taxinomique discutable car chez les bivalves, la forme de la coquille est une adaptation au mode de vie (Cope, 1996a); en outre, des comparaisons de coquilles de dimensions si différentes, peuvent paraître fragiles et peu convaincantes (Babin, 1991). Il est d'ailleurs symptomatique de constater que sur le même critère morphologique, Fordilla qui est désormais comparé à Modiolodon fut initialement rapproché des cycloconchides (Pojeta, 1975, p. 373).

Plus récemment enfin, Waller (1990) a regroupé, d'après les microstructures coquillières, les deux genres Fordilla et Pojetaia en un ensemble monophylétique, dit Fordilloida (ordre créé par Pojeta, 1975), groupe éteint sans descendance selon lui. Runnegar et Pojeta (1992), adoptant ce regroupement, considèrent, en revanche, les fordillides comme des paléotaxodontes ancestraux; Cope (1997), Cope et Babin (1999), Hinz-Schallreuter (2000) se sont rangés à cet avis tandis que Elicki (1994) ou Morton (1996) ont maintenu Fordilla dans les mytilö̈des.

L'appartenance à la classe Bivalvia des genres décrits dans le Cambrien inférieur de Chine par Zhang (1980) a été contestée (Runnegar et Bentley, 1983). Ils sont considérés comme des sténothécoïdes par Runnegar et Pojeta (1992), puis comme des brachiopodes inarticulés déformés par Geyer et Streng (1998) et par Pojeta (2000) qui donne des photographies de Hubeinella, de Xianfengoconcha et de Praelamellodonta. Les hésitations qui demeurent à leur sujet sont néanmoins soulignées par le fait qu'Amler (1999) dans sa classification des bivalves retient, en position systématique indéterminée, la superfamille Praelamellidontoidea Zhang, 1980. D'autres genres, chinois, Oryzoconcha et Jellia, et sibérien, Buluniella, sont assimilés à Pojetaia pour les premiers et à Fordilla pour le troisième par Hinz-Schallreuter (2000) tandis que Cycloconchoides pourrait être un conchostracé branchiopode selon Pojeta (2000). 
En revanche, le statut de Yangtzedonta décrit par Yu (1985) demeure problématique. Connu par une unique valve gauche, ce minuscule fossile présente un contour vaguement ptériomorphoïde et une curieuse charnière pourvue d'une petite barre (dent lamellaire?) et d'une fossette allongée sus-jacente. Cette structure n'est pas sans suggérer quelque rapprochement avec une dent postérieure primaire comme il en est dans les premiers stades ontogénétiques de ptériomorphes (voir Johnston, 1991). Mais il reste malaisé de décider de la signification de ce petit fossile; s'il était véritablement un mollusque bivalve, il fournirait la preuve de la présence de divers types de dentures dès le Cambrien inférieur.

Le genre Modiolopsis lui-même a été cité dans le Cambrien inférieur. A Terre-Neuve, Matthew (1899) signala Modiolopsis thecoides. La description et la figuration données par l'auteur d'une unique valve droite sont, en réalité, très sommaires. Le rapprochement avec Camya, présenté avec réserve par Hinz-Schallreuter (2000), ne paraît pas injustifié pour ce qui concerne le contour de la valve et la denture très simple; il s'accorde moins, en revanche, pour les dimensions puisque Camya est représenté par des coquilles de longueur inférieure à 2 $\mathrm{mm}$ tandis que la forme de Terre-Neuve atteint $9 \mathrm{~mm}$. Le statut exact de ce fossile canadien reste donc ambigu et, sans sa révision (mais le type semble être perdu) l'on ne peut totalement exclure son appartenance au genre Modiolopsis. En 1902, Matthew signala, dans le Cambrien de Cap-Breton, un autre Modiolopsis (?) cf. solvensis Hicks; ce fossile, non figuré, ne peut être pris en compte. Quant au "Modiolopsis" bocagei indiqué par Delgado (1904) dans le Cambrien inférieur du Portugal, il doit être un brachiopode déformé selon Pojeta (2000) qui en fournit une photographie.

$\mathrm{Au}$ Cambrien moyen, trois genres ont été décrits. Ce sont Tuarangia McKinnon, 1982 de Nouvelle-Zélande et de Bornholm (Danemark), Camya Hinz-Schallreuter, 1995 de Bornholm et Arhouriella Geyer et Streng, 1998 de l'Anti-Atlas (Maroc). Le premier de ces genres, initialement assigné aux ptériomorphes par McKinnon (1982), a été considéré comme un rostroconche (Runnegar, 1983; Runnegar et Bentley, 1983; Runnegar et Pojeta, 1992) puis interprété comme un paléotaxodonte (Cope et Babin, 1999; Hinz-Schallreuter, 2000). Le statut de Camya reste problématique et Hinz-Schallreuter $(1995,2000)$ le place dans un ordre indéterminé au sein des paléotaxodontes; mais sa denture réduite pourrait inciter, selon cet auteur, à le considérer comme un représentant ancestral des paléohétérodontes. Quant à Arhouriella, connu seulement par deux valves, sa charnière qui porte un ligament amphidète et deux dents en fait une forme aux affinités énigmatiques. Il convient, enfin, de rappeler, à titre anecdotique, que Fraipont (1910) décrivit un Modiolopsis? malaisii dans le Cambrien moyen de Belgique. Cet échantillon s'est révélé n'être qu'un nodule (Babin, 1993b).

Dans le Cambrien supérieur, l'absence jusqu'alors de récoltes de bivalves constitue un sérieux handicap pour reconstituer l'enchaînement des formes primitives. La datation par conodontes du Cambrien supérieur d'un bloc erratique d'Allemagne qui a livré un exemplaire de Tuarangia (Berg-Madsen, 1987) reste contestable selon Hinz-Schallreuter (1995, 2000).

Les récoltes sont aussi rarissimes dans l'Ordovicien basal (Tremadoc). En Argentine, Harrington (1938) décrivit deux paléotaxodontes, Ctenodonta famatinensis et Palaeoneilo iruyensis dont les dentures ne sont pas observables et qui n'ont pu être révisés depuis (comm. email du 19 octobre 2000 de T. Sánchez). Harrington cita aussi un modiomorphide, Cosmogoniophorina tenuicostata, que Pojeta (1971, p. 17) puis Pojeta et Gilbert-Tomlinson (1977, p. 11) ont interprété comme pouvant être un paléotaxodonte, ce qu'admet, au moins partiellement, Cope (2000, p. 85). Mais Sanchez (1997), qui a retrouvé depuis cette forme dans l'Arenig moyen d'Argentine, considère qu'elle est un "modiomorphoïde" bien que les caractères de la charnière demeurent inconnus.

Une mention particulière doit être accordée à la description faite par Termier et Termier (1971) du paléotaxodonte Afghanodesma pour lequel divers auteurs dont Waller (1998, p. 19) ont depuis regretté de n'avoir pas de renseignements plus précis que les schémas jugés frustes qui en furent fournis initialement. Ces fossiles afghans n'ont, malheureusement, pas pu être revus depuis car il n'a pas été possible de les localiser dans aucune collection publique et ils semblent devoir être considérés comme définitivement perdus. Il convient de noter, en outre, que l'âge d'Afghanodesma demeure problématique. Les spécimens provenaient d'un ensemble de " schistes et grès de couleur rouille " attribué au Tremadoc sans preuves paléontologiques; Desparmet et Montenat (1972), qui admettaient que la "série n'a pu être encore datée avec précision", ajoutaient "les affinités d'Afghanodesma plaident en faveur d'un âge anté-arenigien; d'ailleurs conforme à la position stratigraphique", ce qui relève, évidemment, un peu du raisonnement circulaire pour dater Afghanodesma.

En Australie, Pojeta et Gilbert-Tomlinson (1977) ont signalé dans les Grès de Pacoota, désormais rapportés au Tremadoc supérieur, un paléotaxodonte (Deceptrix? sp. A) avec un unique exemplaire sans denture observable, deux ptériomorphes arcoïdes cyrtodontidés (Cyrtodontula et Pharcidoconcha) et deux "modiomorphides" colpomyidés (Colpantyx et Xestoconcha). En France, (Babin, 1982), a confirmé dans le Tremadoc supérieur de la Montagne Noire, la présence de Babinka indiquée dès 1935 par Thoral.

Ajoutons pour comprendre certaines citations anciennes relatives aux faunes du "Cambrien supérieur" que les stratigraphes britanniques ont longtemps placé le Tremadoc au sommet du Cambrien. Cela ajoute à la confusion et explique, par exemple, qu'Allen et Sanders (1969, p. 392) aient indiqué que les premiers bivalves fossiles sont des Ctenodonata (sic) trouvés dans le Cambrien supérieur en se référant à Cox (1959). Cet auteur se fondait, en réalité, sur les fossiles signalés dans le Tremadoc d'Argentine par Harrington (1938). Cox concluait par ailleurs (1959, p. 202) que les bivalves n'étaient probablement pas présents au Cambrien inférieur et moyen. 
Après cette apparente indigence trémadocienne, le registre fossile montre, en revanche, que la diversification des bivalves est accomplie dans la partie supérieure de l'Ordovicien inférieur (Arenig) puisque tous les superordres sont désormais représentés dans les mers des plates-formes périgondwaniennes. Cela correspond dès lors à une grande diversité des dentures (Fig. 3). La monophylie des bivalves paraissant indiscutable, on peut admettre une diversification très précoce, antéarenigienne, des dentures.

En Chine, Hsü (in Hsü et Ma, 1948) décrivit un Modiolopsis parallelus que Pojeta et Gilbert-Tomlinson (1977, p. 22) ont proposé de placer dans leur nouveau genre de ptériomorphe cyrtodontidé Pharcidoconcha; l'âge exact (Tremadoc? Arenig?) de ce fossile chinois reste, en outre, indéterminé. Enfin, j'ai indiqué précédemment que le Cosmogoniophorina cité autrefois par Harrington dans le Tremadoc d'Argentine est probablement bien un "modiomorphoïde". On constate donc, en définitive, que si la présence de Modiolopsis peut être affirmée dès l'Arenig inférieur (Babin, 1982; Cope, 1996b) et moyen (M. aragonensis nov. sp.), le genre semble avoir eu des représentants dès le Tremadoc.

\section{RELATIONS PALÉOTAXODONTES- PALÉOHÉTÉRODONTES}

Il est remarquable que les bivalves primitifs à denture réduite sont généralement attribués aux paléohétérodontes dont relèvent les actinodontoïdes et parmi lesquels ont été souvent placés les modiomorphoïdes. Camya dès le Cambrien moyen pourrait, selon Hinz-Schallreuter (2000), être une forme ancestrale des paléohétérodontes. Au Tremadoc, le cas de Babinka est caractéristique des difficultés rencontrées pour interpréter les bivalves primitifs. Décrit par Barrande (1881) dans l'Ordovicien moyen de Bohême, ce genre n'est pas aussi rare que l'indiquait McAlester (1964, 1966) puisque, reconnu dès le Tremadoc en Montagne Noire (Thoral, 1935; Babin, 1982), il a été retrouvé dans l'Arenig inférieur au Maroc (Babin et Destombes, 1992), dans l'Arenig supérieur en Suède (Soot-Ryen, 1969) puis dans l'Ordovicien moyen d'Espagne (Babin et Gutiérrez-Marco, 1991) et du Pays de Galles (Cope, 1999). L'historique des diverses interprétations qui en ont été données fut fourni par Babin (1977) qui le considéra comme un actinodontoïde, position généralement admise depuis (Cope, 1997, 1999). Il est intéressant de rappeler néanmoins que Thoral (1935) le classait dans les nuculoïdes et que Pojeta (1978, p. 242), qui considérait Babinka comme un lucinoïde primitif, suggéra qu'il pourrait avoir été, avec sa denture réduite, un descendant direct d'un fordillide. Au Tremadoc sont aussi reconnus, je l'ai indiqué, des "modiomorphoïdes" en Argentine et en Australie.

Dans cette diversification initiale des dentures, on retrouve finalement le problème ancien des relations entre dentures paléotaxodonte et paléohétérodonte. La denture paléotaxodonte représente-t-elle un caractère primitif et l'actinodontie un caractère évolué ou viceversa ? On sait que les opinions ont été très partagées à ce sujet (voir Sánchez et Babin, 1998) et qu'il est maintenant plutôt soutenu que l'habitus paléotaxodonte correspond au mode initial (Cope, 2000).

Les Palaeotaxodonta ont été généralement définis par leur denture taxodonte (Newell, in Moore, 1969, p. N 227) qui est elle-même caractérisée par une série de dents

Figure 3. Bilan provisoire des genres de bivalves du Paléozoïque inférieur (l'échelle verticale est proportionnelle aux durées estimées du Cambrien inférieur à l'Arenig). Les genres cités entre parenthèses sont proches de ceux qui sont représentés. Dans le Cambrien inférieur, le statut de mollusque bivalve des genres Praelamellodonta et Yangtzedonta est parfois contesté. Dans le Tremadoc, l'âge d'Afghanodesma demeure douteux. Les genres trémadociens représentés sont tous du Tremadoc supérieur, le schéma montre donc l'importante lacune de la documentation (Cambrien supérieur et Tremadoc inférieur, soit environ $20 \mathrm{Ma}$ ). Ces faunes trémadociennes de bivalves paraissent dominées par paléohétérodontes et ptériomorphes. Les genres arenigiens sont de l'Arenig inférieur ou moyen; la diversité souligne le caractère apparemment explosif de la radiation ordovicienne dans laquelle les "actinodontes" semblent dominants. La partie noire derrière l'umbo de Catamarcaia correspond à un ligament duplivinculaire. Le type ancestral hypothétique ou protérodonte pourrait avoir été édentule, un habitus présent dès le Tremadoc dans le registre fossile. Il n'est pas fait d'esquisse des relations phylogénétiques mais la distribution suggère une séparation précoce, au Cambrien inférieur, des paléotaxodontes et des "actinodontes" à partir de ce type ancestral.

Provisional knowledge of the genera of bivalves of the Lower Palaeozoic (the vertical spacing is proportional to the duration from the Lower Cambrian to the Arenig). The genera in brackets are close to those figured. Regarding the Lower Cambrian genera, the status of Bivalvia remains questionable for Praelamellodonta and Yangtzedonta. The assignment of Afghanodesma to Tremadoc is dubious. All the Tremadocian figured genera are from the Upper Tremadoc, so that the important break in the record (Upper Cambrian and Lower Tremadoc, about 20 my) is conspicuous. These Tremadocian bivalve faunas seem dominated by palaeoheterodonts and pteriomorphs. The Arenigian genera are from Lower and Middle Arenig; their diversity emphasises the explosive aspect of the Ordovician radiation with an apparent prevalence of the "actinodonts". A duplivincular ligament occupied the black space along the hinge of Catamarcaia. The hypothetical ancestor or proterodont could have been edentulous, a habitus existing from the Tremadoc in the fossil record. No suggestion of the phyletic relations is proposed but the distribution suggests an early separation of palaeotaxodonts and "actinodonts" from the ancestral type, during the earliest Cambrian. 


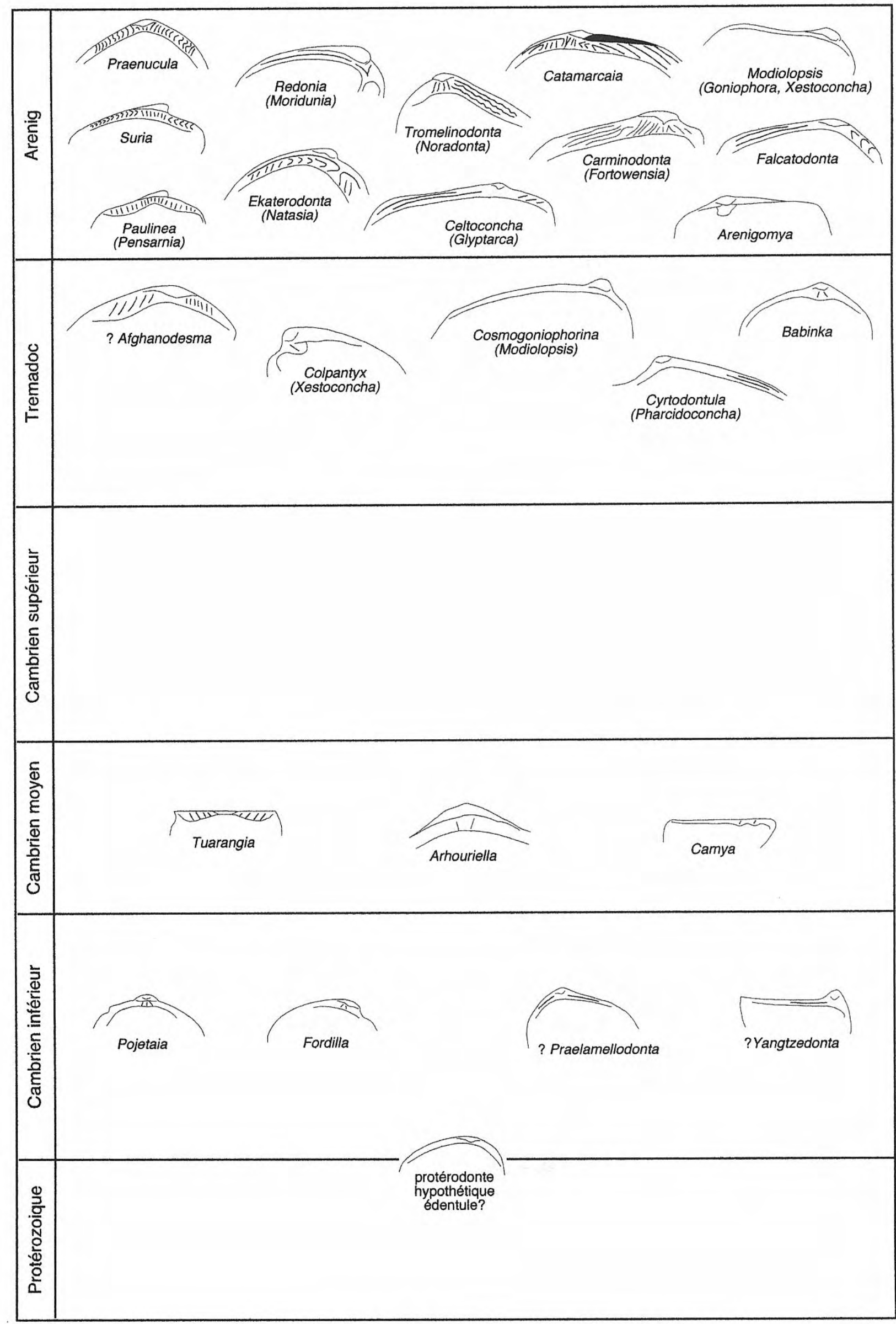


courtes, droites ou en chevrons, occupant toute la longueur du bord dorsal ou interrompue sous le crochet par un résilifer (Cox in Moore, 1969, p. N51). Pourtant la définition des Palaeotaxodonta s'élargit parfois et pour Pojeta (1987, p. 424) si la plupart ont des dents taxodontes, certains sont édentules et quelques-uns ont une denture actinodonte. Mais de façon un peu contradictoire, l'auteur (p. 426) retient néanmoins la denture taxodonte comme l'une des caractéristiques fondamentales du groupe. Allen et Sanders (1969) avaient suggéré que chez Nucinella, un protobranche actuel, la denture serait de type actinodonte; mais Waller (1990) pense qu'elle "est de type paléotaxodonte comme chez les autres protobranches" et Cope (1997) est aussi de cet avis.

Une autre caractéristique des paléotaxodontes actuels, en effet, réside en la possession de branchies constituées de filaments foliacés, type d'organisation dit protobranche et considéré généralement, quoique sans preuves dirimantes, comme le plus primitif. Il en résulte que les paléotaxodontes fossiles sont généralement assimilés aux protobranches tandis que les actinodontes sont considérés comme des filibranches. Néanmoins certains auteurs admettent que les actinodontes sont issus d'un stock de paléotaxodontes ordoviciens qui auraient déjà réalisé la branchie filibranche. Ainsi pour Waller (1988, 1998), un tel paléotaxodonte filibranche pourrait avoir été Tironucula, ce serait Cardiolaria pour Cope (1996a).

Comme on le constate, un certain nombre d'ambiguïtés de terminologie conduit à un imbroglio assez confus résultant de la conception que l'on se fait des rapports phylétiques des paléotaxodontes et des actinodontes. Les dentures sont, il est vrai, des plus intéressants parmi les quelques caractères disponibles sur les bivalves fossiles. Leur utilisation à des fins phylogénétiques se trouve ainsi justifiée dans la pratique sans que l'on puisse affirmer pourtant que ce caractère a une valeur déterminante pour la reconstitution des enchaînements des différents groupes.

Quoi qu'il en soit, l'interprétation des relations phylétiques de ces deux types de dentures demeure largement hypothétique:

- n'est-on pas en présence, dès le Cambrien inférieur et moyen, de formes à dentures différentes?

- doit-on considérer comme de véritables taxodontes des formes (Fordilla, Pojetaia) dont la denture se réduit à un très petit nombre de dents cardinales (prétaxodontes de Carter et al., 2000, p. 53)? On peut certes arguer avec Runnegar et Bentley (1983) que ce faible nombre de dents relève des dimensions minuscules de la coquille, mais on constate que la charnière primitive des larves pélagiques de certains bivalves actuels, plus petites encore, présente souvent un nombre plus élevé de denticules (voir Babin et Le Pennec, 1982, par exemple); il en est de même pour des nuculoïdes actuels (Carter et al., 2000).

- n'a-t-on pas dès le Tremadoc et à l'Arenig inférieur, avec l'active diversification ordovicienne des bivalves, une panoplie de types de dentures parmi lesquels le type édentule est représenté par Modiolopsis notamment (Fig. 3)?

- ne pourrait-on dès lors concevoir que les formes ancestrales des bivalves ont pu être, au Protérozoïque supérieur (Vendien) ou au Cambrien basal (NemkytDaldinien) de petites coquilles à charnière édentule? La simplicité d'une telle denture ne serait pas incompatible avec un mode de vie dans la méiofaune.

A partir d'un tel stock initial, qui reste jusqu'alors non documenté il est vrai, les types paléotaxodonte et paléohétérodonte initiaux pourraient s'être réalisés indépendamment, rendant injustifiées et inutiles les discussions sur l'antériorité de l'un ou de l'autre de ces types. C'est ce que voulaient suggérer Sánchez et Babin (1998) en se demandant si les discussions sur l'antériorité des paléotaxodontes ou des actinodontes ne relèvent pas d'un faux problème.

A ce point de notre propos, il est intéressant de considérer les arbres phylogénétiques présentés dans la littérature. On constate que certains d'entre eux semblent impliquer une cladogenèse rapide des types paléotaxodonte et actinodonte à partir d'un type ancestral commun non connu.

Ainsi Zhang (1980, p. 16) envisageait-il, sur la base il est vrai d'un bivalve contesté, Praelamellodonta, une divergence précoce des deux types dentaires.

Pojeta (1987, p. 422-423), qui considérait alors Pojetaia comme un paléotaxodonte et Fordilla comme un isofilibranche, illustre une phylogénie qui renvoie implicitement à un ancêtre précambrien inconnu.

L'arbre de Runnegar et Pojeta (1992, p. 120), désormais obsolète eu égard à la signification accordée à Tuarangia et à Pseudomonya, évoquait un ancêtre bivalve commun à deux types de charnières, l'un à dents cardinales, l'autre à dents latérales.

Le schéma de Morton (1996, p. 348) suggère un ancêtre antécambrien commun aux deux rameaux, celui des paléotaxodontes et celui des autres bivalves.

Waller $(1990,1998)$ a présenté d'intéressantes reconstitutions phylogénétiques pour les bivalves. Dans son arbre de 1990 (p. 62), les apomorphies III d'une forme hypothétique ancestrale des protobranches et des autobranches sont "ligament fibreux et denture paléotaxodonte". Mais l'auteur postule aussi l'existence antérieure d'un proto-bivalve non nommé et l'on pourrait interpréter celui-là comme une forme bivalve primitive, pouvant avoir été édentule. Dans son arbre phylétique des mollusques (Waller, 1998, p. 2), la liste (p. 10) des apomorphies du noeud 10 (Bivalvia) ne mentionne pas de caractères dentaires; il en est de même pour le nœud PR-1 de l'arbre des Protobranchia (p. 17). Parmi les apomorphies des Autobranchia (nœud AB-1, p. 20 et Figure 4, p. 22), l'auteur indique une denture taxodonte modifiée ou une ontogenèse dentaire de type umburride (définie par Johnston, 1991). Waller, qui privilégie ainsi une descendance paléotaxodonte-actinodonte, tente de reconstituer des étapes de cette évolution en utilisant des genres connus de l'Ordovicien (1998, Fig. 5, p. 33). Mais les divergences se sont réalisées avant l'apparition des formes arenigiennes utilisées dans ce schéma puisqu'au 
Tremadoc déjà s'observe une notable diversité des dentures avec divers types de paléohétérodontes (Babinka, Colpantyx, Xestoconcha) et de ptériomorphes (Cyrtodontula, Pharcidomorpha).

Hinz-Schallreuter confronte (2000, Fig. 4, p. 231) deux arbres possibles de relations pour les premiers bivalves. Elle préfère adopter, dit-elle, celui dans lequel tous les bivalves cambriens sont considérés comme des paléotaxodontes mais le statut dentaire de Camya intégré dans ce schéma demeure, nous l'avons noté, quelque peu ambigu.

Le cladogramme de Carter et al. (2000, Fig. 4, p. 58) indique un statut de groupes-frères pour les Palaeotaxodonta et les Autobranchia (Heteroconchia, Anomalodesmata et Pteriomorphia) avec un ancêtre commun (noeud 7). Dans leurs commentaires, ces auteurs suggèrent un ancêtre commun édentule pour Tuarangia et Pojetaia (p. 60) et considèrent, par ailleurs, que la denture primitive précédant la divergence des Palaeotaxodonta et des Autobranchia n'était ni paléotaxodonte ni actinodonte (p. 61).

L'attribution d'une antériorité aux paléotaxodontes dans la phylogénie relève donc davantage d'un choix que d'une démonstration car l'attribution aux paléotaxodontes de l'ensemble des bivalves du Cambrien reste très conjecturale.

D'autres arguments peuvent certes être avancés en faveur d'une telle antériorité des paléotaxodontes:

- Waller (1998, p. 33) pense que la nature du système ligamentaire et la position de l'insertion de l'adducteur antérieur permettent de relier les tironuculidés aux autobranches suggérant ainsi que la denture taxodonte est primitive.

- La coquille larvaire de bivalves actuels de divers groupes qui montre une charnière pourvue de plusieurs petites dents pourrait être interprétée comme une récapitulation ontogénétique de la phylogenèse de ces groupes (Babin et Le Pennec, 1982), ce qui suggérerait aussi que le type paléotaxodonte est ancestral.

Cependant, la denture paléotaxodonte typique avec son ensemble parfois complexe de nombreuses dents, représentée dès l'Ordovicien inférieur, correspond à une spécialisation de la charnière et marque donc déjà un degré notable d'évolution. Il en est de même pour la denture actinodonte (paléohétérodonte) aux motifs très variés elle aussi à l'Ordovicien inférieur.

En définitive, prenant acte de notre ignorance actuelle sur les débuts de l'histoire de la classe des bivalves, il peut paraître raisonnable de désigner d'un terme provisoire, protérodonte par exemple, le type-souche hypothétique à partir duquel se seraient réalisées ultérieurement et indépendamment les premières dentures paléotaxodonte, actinodonte et ptériomorphe véritables. Ce type ancestral, qui demeure inconnu et conjectural, pourrait avoir été soit édentule, soit pourvu d'une denture si fruste (simples denticules cardinaux réduits ou simples bourrelets d'articulation évoquant des dents latérales) qu'elle ne pourrait être valablement qualifiée de paléotaxodonte ou d'actinodonte. La réalisation des branchies filibranches dont Cope (1996a) considère qu'elle a été le facteur déterminant de la diversification ordovicienne des bivalves, pourrait s'être faite aussi précocement chez des formes primitives à charnière non paléotaxodonte lors des premières divergences.

La sagesse d'une telle position d'attente, qui suggère notamment que paléotaxodontes et paléohétérodontes pourraient être des groupes-frères, incite, quoi qu'il en soit, à poursuivre intensément les investigations dans le Cambrien basal et le Protérozoïque terminal.

\section{REMERCIEMENTS}

Les photographies ont été réalisées par N. Podevigne (Université Claude Bernard-Lyon I) que nous remercions. L'un de nous (C.B.) est redevable à F. Doré qui lui a confié pour examen son matériel du Cambrien de Normandie ainsi qu'à tous ceux qui lui ont fourni divers renseignements, paléontologiques ou stratigraphiques, J. Blaise (Nantes), E. Liñán (Zaragoza), C. Montenat (Paris), I. Rábano (Madrid). Il remercie également ceux qui ont bien voulu l'aider dans l'enquête menée, sans succès, pour retrouver Afghanodesma dans des collections publiques, A. Lauriat-Rage (Paris), M. Philippe et A. Prieur (Lyon). Enfin, il est redevable aux deux rapporteurs, J.C.W. Cope et T.M. Sánchez, de leurs remarques et suggestions qui ont contribué à améliorer la présentation et le contenu de cet article, étant entendu qu'il assume seul la responsabilité des propositions et choix présentés ici.

\section{RÉFÉRENCES BIBLIOGRAPHIQUES}

Allen, J.A. and Sanders, H.L. 1969. Nucinella serrei Lamy (Bivalvia : Protobranchia), a monomyarian solemyid and possible living actinodont. Malacologia, 7, 381-396.

Amler, M.R.W. 1999. Synoptical classification of fossil and Recent Bivalvia. Geologica et Palaeontologica, 33, 237248.

Babin, C. 1966. Mollusques Bivalves et Céphalopodes du Paléozö̈que armoricain. Etude systématique. Essai sur la phylogénie des Bivalves. Esquisse paléoécologique. Imprimerie Commerciale et Administrative, Brest. 479 pp.

Babin, C. 1977. Etude comparée des genres Babinka Barrande et Coxiconcha Babin (Mollusques bivalves de l'Ordovicien). Intérêt phylogénétique. Geobios, 10, 5179.

Babin, C. 1982. Mollusques bivalves et rostroconches. In: Brachiopodes (Articulés) et Mollusques (Bivalves, Rostroconches, Monoplacophores, Gastropodes) de l'Ordovicien inférieur (Tremadocien-Arenigien) de la Montagne Noire (France méridionale) (Eds. C. Babin, R. Courtessole, M. Mélou, J. Pillet, D. Vizcaïno et E.L. Yochelson). Mémoires de la Société des Etudes Scientifiques de l'Aude, Carcassonne, 37-49.

Babin, C. 1991. De la morphologie à la morphogenèse : un champ ouvert aux investigations paléontologiques. Geobios, M.S. n 13, 9-12. 
Babin, C. 1993a. Rôle des plates-formes gondwaniennes dans les diversifications des mollusques bivalves durant l'Ordovicien. Bulletin de la Société géologique de France, 164, 141-153.

Babin, C. 1993b. A propos d'un prétendu mollusque bivalve du Cambrien de Belgique. Annales de la Société géologique de Belgique, 116, 13-14.

Babin, C. 1995. The initial Ordovician bivalve mollusc radiations on the western Gondwanan shelves. In: Ordovician Odyssey (Eds. J.D. Cooper, M.L. Droser and S.C. Finney), Pacific Section Society Sedimentary Geology, 77, 491-498.

Babin, C. et Destombes, J. 1992. Les mollusques bivalves et rostroconches ordoviciens de l'Anti-Atlas marocain : Intérêt paléogéographique de leur inventaire. Géologie Méditerranéenne, 17, 243-261 (daté 1990).

Babin, C. and Gutiérrez-Marco, J.C. 1991. Middle Ordovician bivalves from Spain and their phyletic and palaeogeographic significance. Palaeontology, 34, 109-147.

Babin, C. et Le Pennec, M. 1982. Ontogenèse et phylogenèse : A propos de quelques caractères dentaires des mollusques bivalves. Malacologia, 22, 709-720.

Barrande, J. 1881. Système silurien du centre de la Bohême. Vol. 6: Acéphalés. Paris et Prague, 342 pp., 361 pls.

Barrois, C. 1891. Mémoire sur la Faune du Grès armoricain. Annales de la Société géologique du Nord, 19, 134-237.

Berg-Madsen, V. 1987. Tuarangia from Bornholm (Denmark) and similarities in Baltoscandian and Australasian late Middle Cambrian faunas. Alcheringa, 11, 245-259.

Bradshaw, M.A. 1999. Lower Devonian bivalves from the Reefton Group. New Zealand. Association of Australasian Palaeontologists, Memoir 20, 1-171.

Carls, P. 1975. The Ordovician of the Eastern Iberian Chains near Fombuena and Luesma (Prov. Zaragoza, Spain). Neues Jährbuch für Geologie und Paläontologie, Abhandlungen 150 (2), 127-146.

Carter, J.G. and Seed, R. 1998. Thermal potentiation and mineralogical evolution in Mytilus (Mollusca, Bivalvia). In: Bivalves: An Eon of Evolution (Eds. P.A. Johnston and J.W. Haggart). University of Calgary Press, 87-117.

Carter, J.G., Campbell, D.C. and Campbell, M.R. 2000. Cladistic perspectives on early bivalve evolution. In: The Evolutionary Biology of the Bivalvia (Eds. E.M. Harper, J.D. Taylor and J.A. Crame). Geological Society, London, Special Publications, 177, 47-79.

Conrad, T.A. 1838. Monograph of the family Unionidae or Najades of Lamarck (Fresh water bivalve shells). Philadelphia, 1-110, 60 pls.

Cope, J.C.W. 1996a. The early evolution of the Bivalvia. In: Origin and evolutionary radiation of the Mollusca (Ed. J. Taylor). Oxford University Press, 361-370.

Cope, J.C.W. 1996b. Early Ordovician (Arenig) bivalves from the Llangynog Inlier, South Wales. Palaeontology, 39, 979-1025.

Cope, J.C.W. 1997. The early phylogeny of the class Bivalvia. Palaeontology, 40, 713-746.

Cope, J.C.W. 1999. Middle Ordovician bivalves from MidWales and the Welsh Borderland. Palaeontology, 42, 467-499.
Cope, J.C.W. 2000. A new look at early bivalve phylogeny. In: The Evolutionary Biology of the Bivalvia (Eds. E.M. Harper, J.D. Taylor and J.A. Crame). Geological Society, London, Special Publications, 177, 81-95.

Cope, J.C.W. and Babin, C. 1999. Diversification of bivalves in the Ordovician. Geobios, 32, 175-185.

Cox, L.R. 1959. The geological history of the Protobranchia and the dual origin of taxodont Lamellibranchia. Proceedings of the Malacological Society of London, 33, 200-209.

Delgado, J.F.N. 1904. Faune cambrienne du Haut-Alemtejo (Portugal). Comunicacões da Comisão do Servico Geologico de Portugal, 5, 307-374.

Desparmet, R. et Montenat, C. 1972. Les transgressions du Paléozoïque en Hazarajat (Afghanistan central). Revue de Géographie physique et de Géologie dynamique, 14, 397-414.

Doré, F. 1994. Cambrian of the Armorican Massif. In: Premesozoic geology in France and related areas (Ed. J.D. Keppie). Springer Verlag, 136-141.

Elicki, O. 1994. Lower Cambrian carbonates from eastern Germany: Palaeontology, stratigraphy and palaeogeography. Neues Jahrbuch für Geologie und Paläontologie Abhandlungen, 191, 69-93.

Fang, Z. 1998. Revision and taxonomic position of the aberrant Devonian bivalve Beichuania. In: Bivalves: An Eon of Evolution (Eds. P.A. Johnston and J.W. Haggart). University of Calgary Press, 185-192.

Fang, Z. and Morris, N.J. 1997. The genus Pseudosanguinolites and some modioliform bivalves (mainly Palaeozoic). Palaeoworld, 7, 49-74.

Fortey, R.A. and Owens, R.M. 1987. The Arenig Series in South Wales. Bulletin British Museum Natural History (Geology), 41, 69-307.

Fraipont, C. 1910. Modiolopsis ? ? Malaisii, Ch. Fraip., Lamellibranche nouveau du Revinien Belge (Cambrien moyen). Annales de la Société géologique de Belgique, 37, M3-4.

Geyer, G. and Streng, M. 1998. Middle Cambrian pelecypods from the Anti-Atlas, Morocco. Revista Española de Paleontología, $\mathbf{n}^{\circ}$ extr. Homenaje al Prof. Gonzalo Vidal, 83-96.

Grobben, K. 1894. Zur Kenntniss der Morphologie der Verwandschaftsverhältnisse und des Systems der Mollusken. Sitzungsberichte der Kaiserlichen Akademie der Wissenschaften, Mathematisch-Naturwissenschaftlichen Classe, 103, 61-86.

Gutiérrez-Marco, J.C. 1986. Graptólitos del Ordovícico Español. Tesis. Universidad Complutense de Madrid. Facultad de las Ciencias Geológicas. Vol. I : 1-318. (inédit).

Gutiérrez-Marco, J.C., Babin, C. y Porro Mayo, T. 1997. Moluscos bivalvos de las facies cuarcíticas del Ordovícico Inferior centroibérico. Geogaceta, 22, 85-88.

Hall, J. 1847. Palaeontology. Geological Survey of New York, I-XXIII + 1-338, 87 pls.

Hammann, W., Robardet, M. and Romano, M. 1982. The Ordovician System in Southwestern Europe (France, Spain and Portugal). International Union of Geological Sciences, 11, 1-47. 
Harrington, H.J. 1938. Sobre las faunas del Ordoviciano Inferior del Norte Argentino. Revista del Museo de La Plata (Nueva Serie), 1, Sección Paleontología, 4, 109-289.

Hinz-Schallreuter, I. 1995. Muscheln (Pelecypoda) aus dem Mittelkambrium von Bornholm. Geschiebekunde aktuell, 11, 71-84.

Hinz-Schallreuter, I. 2000. Middle Cambrian Bivalvia from Bornholm and a review of Cambrian bivalved Mollusca. Revista Española de Micropaleontología, 32, 225-242.

Hsü, S.C. and Ma, C.T. 1948. The I-Chang Formation and the Ichangian fauna. National Research Institute of Geology, Academia Sinica, Contribution 8, 1-51.

Isberg, O. 1934. Studien über Lamellibranchiaten des Leptaenakalkes in Dalarna. Hakan Ohlssons Buchdruckerei, Lund, 1-492.

Jell, P.A. 1980. Earliest known pelecypod on Earth - a new Early Cambrian genus from South Australia. Alcheringa, 4, 233-239.

Johnston, P.A. 1991. Systematics and ontogeny of a new bivalve, Umburra cinefacta, from the Silurian of Australia: implications for pteriomorphian evolution. Alcheringa, 15, 293-319.

Johnston, P.A. 1993. Lower Devonian Pelecypoda from southeastern Australia. Memoir of the Australasian Association, 14, 1-134.

Johnston, P.A. 1996. Middle Ordovician (Caradoc) pelecypods from the Advance Formation, British Columbia. Geological Survey of Canada Bulletin, 491, 113-129.

Khalfin, L.L. 1958. Mollusques lamellibranches de l'Ordovicien du Tchou Ilyiskikh Gor. In: Ordovik Kazakhstana, III. Akademia Nauk SSSR, Trudy Geologitcheskogo Instituta, 9, 139-196 (en russe).

Krasilova, I.N. 1979. Mollusques bivalves des horizons Mangazeisky, Dolborsky et Ketsky. In: Fauna ordovika sredneï Sibiry (Eds. Kh.S. Rozman, G.A. Stukalina, I.N. Krasilova et V.A. Sytova Akademia). Nauk SSSR, Ordena Trudovogo Krasnogo Znameni Geologitcheskï Institut, Trudy 330, 79-130 (en russe).

Liljedahl, L. 1994. Silurian nuculoid and modiomorphid bivalves from Sweden. Fossils \& Strata, 33, 1-89.

Matthew, G.F. 1899. Preliminary notice of the Etcheminian fauna of Newfoundland. Bulletin of the Natural History Society of New Brunswick, 4, 189-209.

Matthew, G.F. 1902. Additional notes on the Cambrian of Cape Breton with description of new species. Bulletin Natural History Society of New Brunswick, 20, 377-425.

McAlester, A.L. 1964. Transitional Ordovician bivalve with both monoplacophoran and lucinacean affinities. Science, 146, 1293-1294.

McAlester, A.L. 1966. Evolutionary and systematic implications of a transitional Ordovician lucinoid bivalve. Malacologia, 3, 433-439.

McKinnon, D.I. 1982. Tuarangia paparua n. gen. and n. sp. A late Middle Cambrian pelecypod from New Zealand. Journal of Paleontology, 56, 589-598.

Mergl, M. and Liñán, E. 1986. Some Cambrian Brachiopoda of the Cordillera Ibérica and their biostratigraphical significance. In: Memorias I Jornadas de Paleontología (Ed. E. Villas), 159-180.
Moore, R.C. (Ed.) 1969. Treatise on Invertebrate Paleontology, Part N, Vol. 1, Mollusca 6, Bivalvia, N1N489.

Morton, B. 1996. The evolutionary history of the Bivalvia. In: Origin and evolutionary radiation of the Mollusca (Ed. J.D. Taylor). Oxford University Press, 337-359.

Munier-Chalmas, E. et Bergeron, J. 1888. Sur la présence de la faune primordiale (Paradoxidien) dans les environs de Ferrals-les-Montagnes (Hérault). Comptes rendus de l'Académie des Sciences, Paris, 106, 377.

Paris, F. 1990. The Ordovician chitinozoan biozones of the Northern Gondwana Domain. Review of Palaeobotany and Palynology, 66, 181-209.

Paris, F., Robardet, M., Dabard, M.P., Feist, R., Ghienne, J.F., Guillocheau, F., Le Hérissé, A., Loi, A., Mélou, M., Servais, T., Shergold, J., Vidal, M. and Vizcaïno, D. 2000. Ordovician rocks of France. In: Quo vadis Ordovician? (Eds. P. Kraft and O. Fatka). Acta Universitatis Carolinae Geologica, 43, 85-88.

Pojeta, J., Jr. 1971. Review of Ordovician Pelecypods. Geological Survey Professional Paper, 695, 1-46.

Pojeta, J., Jr. 1975. Fordilla troyensis Barrande and the early pelecypod phylogeny. United States Bulletins of American Paleontology, 67, 363-384.

Pojeta, J., Jr. 1978. The origin and early taxonomic diversification of pelecypods. Philosophical Transactions of the Royal Society of London, B 284, 225-246.

Pojeta, J., Jr. 1987. Class Pelecypoda. In: Fossil Invertebrates (Eds. R.S. Boardman, A.H. Cheetham and A.J. Rowell). Blackwell Science, 386-435.

Pojeta, J., Jr. 2000. Cambrian Pelecypoda (Mollusca). American Malacological Bulletin, 15, 157-166.

Pojeta, J., Jr. and Gilbert-Tomlinson, J. 1977. Australian Ordovician pelecypod molluscs. Bureau of Mineral Resources, Geology and Geophysics, Bulletin, 174, 1-64.

Pojeta, J. Jr., and Runnegar, B. 1974. Fordilla troyensis and the early history of pelecypod mollusks. American Scientist, 62, 705-711.

Pojeta, J., Jr. and Runnegar, B. 1985. The early evolution of diasome molluscs. In: The Mollusca, vol. 10 (Eds. E.R. Trueman and M.R.Clarke), Academic Press, Inc., 295336.

Pojeta, J., Jr., Runnegar, B. and Kříž, J. 1973. Fordilla troyensis Barrande: the oldest known pelecypod. Science, 180, 866-868.

Rouault, M. 1850. Note préliminaire sur une nouvelle formation découverte dans le terrain silurien inférieur de la Bretagne. Bulletin de la Société géologique de France (2), 7, 724-744.

Runnegar, B. 1983. Molluscan phylogeny revisited. Memoir of the Association of Australasian Palaeontologists, $\mathbf{1}$, 121-144.

Runnegar, B. and Bentley, C. 1983. Anatomy, ecology and affinities of the Australian Early Cambrian bivalve Pojetaia runnegari Jell. Journal of Paleontology, 57, 7392.

Runnegar, B. and Pojeta, J., Jr. 1992. The earliest bivalves and their Ordovician descendants. American Malacological Bulletin, 9, 117-122. 
Sampelayo, P. H. 1935. Explicación del nuevo Mapa geológico de España a escala 1: 1.000.000. El Sistema Cambriano. Memorias del Instituto Geológico y Minero de España, 41, 291-529.

Sánchez, T. 1997. Additional Mollusca (Bivalvia and Rostroconchia) from the Suri Formation, Early Ordovician (Arenig), western Argentina. Journal of Paleontology, 71, 1046-1054.

Sánchez, T. and Babin, C. 1998. The origin of actinodont from taxodont dentition or vice-versa: an unnecessary controversy? In: Bivalves: an Eon of Evolution (Eds. P.A. Johnston and J.W. Haggart). University of Calgary Press, 409-412.

Seilacher, A. 1982. Distinctive features of sandy tempestites. In: Cyclic and Event Stratification (Eds. G. Einsele and A. Seilacher). Springer, Berlin, Heidelberg, New York, 333-349.

Soot-Ryen, H. 1969. A new species of Babinka (Bivalvia) from the Lower Ordovician of Öland, Sweden. Palaeontology, 12, 173-177.

Termier, G. et Termier, H. 1971. Description. In: Desparmet, R., Termier, G. et Termier, H. Sur un bivalve protobranche anté-arenigien trouvé au nord de Wardak (Afghanistan). Geobios, 4, 146-150.

Thoral, M. 1935. Contribution à l'étude paléontologique de l'Ordovicien inférieur de la Montagne Noire. Imprimerie de la Charité, Montpellier, 1-362.

Tromelin, G. de et Lebesconte, P. 1875. Note sur quelques fossiles des grès siluriens de St-Germain/Ille, La
Bouexière, Champoux, etc. Ed. Cotonnec, Quimper, 1-8.

Waller, T.R. 1988. A phylogeny of Bivalvia: Progress and problems. Program and Abstracts, 54th Annual Meeting, American Malacological Union, Charleston, 19.

Waller, T.R. 1990. The evolution of ligament systems in the Bivalvia. In: Proceedings of a Memorial Symposium in Honour of Sir Charles Maurice Yonge (Ed. B. Morton), Hong Kong University Press, 49-71.

Waller, T.R. 1998. Origin of the molluscan class Bivalvia and a phylogeny of major groups. In: Bivalves: an Eon of Evolution (Eds. P.A. Johnston and J.W. Haggart). University of Calgary Press, 1-45.

Wiemer, B., 1975. Erläuterungen zur geologischen Kartierung des Gebietes SE Atea/Acered in den Westlichen Iberischen Ketten. Diplomarbeit, Institut für Paläontologie, Würzburg Universität, 1-95. (inédit).

Wolf, R. 1980. The lower and upper boundary of the Ordovician system of some selected regions (Celtiberia, Eastern Sierra Morena) in Spain. Part 1: The Lower Ordovician sequence of Celtiberia. Neues Jahrbuch für Geologie und Paläontologie, Abhandlungen 160, 118137.

Yu, W. 1985. Yangtzedonta - a problematic Bivalvia from the Meishucunian Stage, China. Acta Micropalaeontologica Sinica, 2, 401-408.

Zhang, R. 1980. On the earliest bivalve fauna - bivalves from Lower Cambrian Tianheban Formation, Xianfeng, Hubei. Bulletin of the Chinese Academy of Geological Sciences, 7, résumé en anglais, 17-18. 